\title{
Precise Intradermal Injection of Nanofat- Derived Stromal Cells Combined with Platelet-Rich Fibrin Improves the Efficacy of Facial Skin Rejuvenation
}

\author{
Zhi-Jie Liang ${ }^{a} \quad$ Xiang Lu ${ }^{b} \quad$ De-Quan Lic Yi-Dan Liang ${ }^{d}$ Dan-Dan Zhue \\ Fang-Xiao Wu ${ }^{e}$ Xiao-Lin Yie Ning He ${ }^{e}$ Yan-Qing Huang ${ }^{f}$ Chao Tang $^{f}$ \\ Hong-mian Lie
}

aDepartment of Breast and Thyroid Surgery, The Fifth Affiliated Hospital of Guangxi Medical University \& The First People's Hospital of Nanning, Nanning bepartment of Hematology, The Fifth Affiliated Hospital of Guangxi Medical University \& The First People's Hospital of Nanning, Nanning 'Department of Breast Plastic Surgery, The Third Hospital of Nangchang, Nanchang ${ }^{\mathrm{d} C e n t r a l ~ L a b o r a t o r y ~ o f ~ M e d i c a l ~}$ Science, The Fifth Affiliated Hospital of Guangxi Medical University \& The First People's Hospital of Nanning, Nanning eDepartment of Plastic and Aesthetic Surgery, The Fifth Affiliated Hospital of Guangxi Medical University \& The First People's Hospital of Nanning, Nanning fDepartment of Plastic and Aesthetic Surgery, The Mengxiang Plastic Hospital, Nanning, China

\section{Key Words}

Facial skin aging - Rejuvenation - Nanofat-derived stem cells • Platelet-rich fibrin • Stromal vascular fraction

\begin{abstract}
Background/Aims: The rejuvenation properties of nanofat grafting have been described in recent years. However, it is not clear whether the clinical efficacy of the procedure is attributable to stem cells or linked to other components of adipose tissue. In this study we isolated nanofat-derived stem cells (NFSCs) to observe their biological characteristics and evaluate the efficacy of precise intradermal injection of nanofat combined with platelet-rich fibrin (PRF) in patients undergoing facial rejuvenation treatment. Methods: Third-passage NFSCs were isolated and cultured using a mechanical emulsification method and their surface CD markers were analyzed by flow cytometry. The adipogenic and osteogenic nature and chondrogenic differentiation capacity of NFSCs were determined using Oil Red O staining, alizarin red staining, and Alcian blue staining, respectively. Paracrine function of NFSCs was evaluated by enzyme-linked immunosorbent assay (ELISA) at 1, 3, 7, 14, and 28 days after establishing the culture. Then, the effects of PRF on NFSC proliferation were assessed in vitro. Finally, we compared the outcome in 103 patients with facial skin aging who underwent both nanofat and intradermal PRF injection (treatment group) and 128 patients who underwent hyaluronic acid (HA) injection treatment (control group). Outcomes in the two groups were compared
\end{abstract}

Z.-J. Liang and X. Lu contributed equally to this work and share the first authorships.

Hong-mian Li

KARGER
Department of Plastic and Aesthetic Surgery, The Fifth Affiliated Hospital of Guangxi

Medical University \& The First People's Hospital of Nanning, No. 89 Qixing Road,

Qingxiu District, Nanning 530022, (China)

Tel. 8617776022378, Fax 86-0771-2617892,E-Mail 18022081568@163.com 


\section{Cellular Physiology Cell Physiol Biochem 2018;47:316-329

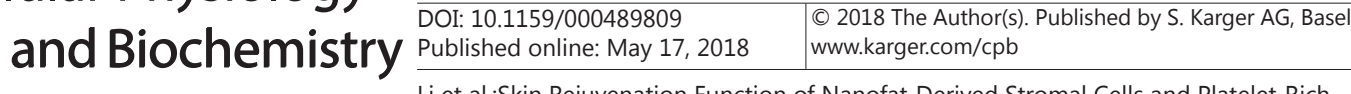 \\ Li et al.:Skin Rejuvenation Function of Nanofat-Derived Stromal Cells and Platelet-Rich \\ Fibrin}

by assessing pictures taken at the same angle before and after treatment, postoperative recovery, incidence of local absorption and cysts, and skin quality before treatment, and at 1 , 12, 24 months after treatment using the VISIA Skin Image Analyzer and a SOFT5.5 skin test instrument. Results: NFSCs expressed CD29, CD44, CD49d, CD73, CD90, and CD105, but did not express CD34, CD45, and CD106. NFSCs also differentiated into adipocytes, osteoblasts, and chondrocytes under appropriate induction conditions. NFSCs released large amounts of growth factors such as VEGF, bFGF, EGF, and others, and growth factor levels increased in a time-dependent manner. At the same time, PRF enhanced proliferation of NFSCs in vitro in a dose-dependent manner, and the growth curves under different concentrations of PRF all showed plateaus $6 \mathrm{~d}$ after seeding. Facial skin texture was improved to a greater extent after combined injection of nanofat and PRF than after control injection of HA. The nanofatPRF group had a higher satisfaction rate. Neither treatment caused any complications such as infection, anaphylaxis, or paresthesia during long-term follow-up. Conclusion: NFSCs demonstrate excellent multipotential differentiation and paracrine function, and PRF promotes proliferation of NFSCs during the early stage after seeding. Both nanofat-PRF and HA injection improve facial skin status without serious complications, but the former was associated with greater patient satisfaction, implying that nanofat-PRF injection is a safe, highly effective, and long-lasting method for skin rejuvenation.

\section{Introduction}

Aging is an intrinsic event in humans that is influenced by a variety of factors, including biological, genetic, and environmental factors. Facial skin aging is the most visible manifestation of aging, resulting in physical, physiological, psychological, and social changes. There are also obvious clinically designated cutaneous effects such as peculiarity of uneven skin color, wrinkles, sunspots, and sagging skin, which often vary based on ethnic origin. Many other factors affect the aging process, such as inherited genetic traits, internal environment homeostasis, hormonal status, working conditions, and climatic, social, and cultural conditions.

Intradermal injection of small-molecule hyaluronic acid (HA) has been widely used to treat aging facial skin by replenishing the HA component of the dermis. However, its therapeutic effects are not maintained over time, because it has an absorbable micromolecular structure. Autologous structural fat transplant is traditionally used for treating facial soft tissue depressions [1-3], and in addition to improving facial structure, it notably improves skin condition.

In 2013, Tonnard et al. reported on the efficiency and safety of treating skin aging with nanofat, which is different from traditional fat transplant, using intradermal injection of an emulsified fat suspension to promote skin rejuvenation [4]. Our previous research found that numerous mesenchymal stem cells (MSCs) were present in an isolated culture of nanofat and were designated nanofat-derived stem cells (NFSCs).

NFSCs belong to a subcategory of adipose-derived stem cells (ASCs), which are known to participate in repair after injury, and their functions in skin rejuvenation have been described [5]. Regeneration of collagen, elastin, extra cellular matrix, and microcirculation is crucial for reversing the signs of skin aging. An observational study of histologic and ultrastructural changes in skin after injection of fat grafts and ASCs revealed that this treatment was effective in modifying the structure of the dermis for the purpose of rejuvenation [5].

Multiple differentiation and paracrine functions of ASCs contribute to their tissuerepairing properties, which are improved by stimulation with platelet-rich fibrin (PRF) [6, 7]. PRF is also a known skin rejuvenator that has been used in esthetic medical procedures for anti-aging effects in skin [8]. Despite the efficacy of fat transplants and HA injection for skin rejuvenation, a less invasive and more effective approach is always preferred. The purpose of this study was to determine the efficacy and safety of using a nanofat-PRF mixture in a novel injection procedure for skin rejuvenation. 


\section{Cellular Physiology Cell Physiol Biochem 2018;47:316-329

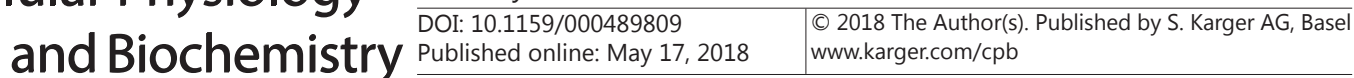 \\ Li et al.:Skin Rejuvenation Function of Nanofat-Derived Stromal Cells and Platelet-Rich Fibrin}

\section{Materials and Methods}

\section{Patient consent and ethical approval}

This study was approved by the Research Ethics Committee of the Fifth Affiliated Hospital of Guangxi Medical University on August 10, 2014. In total, 103 patients (test group) with aging facial skin were selected at random for inclusion. Consultations and diagnosis were held in the Plastic and Aesthetic Surgery Department of the Fifth Affiliated Hospital of Guangxi Medical University and the First People's Hospital of Nanning and at the Nanning Dream Plastic and Aesthetic Hospital between October 2014 and October2016. Patients were 12 men and 91women aged 24 and 55 years (mean age: 28.5 years). All patients had skin types of III or IV. In addition, 128 age-, sex-, and symptom-matched control subjects were enrolled over the same period and underwent HA injection. Pre- and post-injection photos were taken of each patient, and all patients provided written informed consent.

\section{Extraction and preservation of PRF}

PRF was prepared as previously reported [9]. Briefly, $20 \mathrm{~mL}$ of venous blood was collected from patients during liposuction, into 4 glass-coated, aseptic, negative-pressure plastic tubes without anticoagulant. After centrifugation at $2700 \mathrm{rpm} / \mathrm{minute}$ for $12 \mathrm{~min}$ followed by a 5-min rest period, the blood was separated into three layers: the top layer, which consisted of transparent yellow liquid, was platelet-poor plasma (PPP); the middle layer, which consisted of faint yellow gelatinous matter, was the PRF membrane (Fig. 1A); and the bottom layer, which consisted of rufous matter, was erythrocyte fragments. The supernatant was discarded, PRF was collected, and the fibrous membrane was then prepared. The red ends of the PRF contain large amounts of cell factors, so they were cut off lengthwise to ensure that they were well distributed. We prepared three different proliferation media (DMEM, $100 \mathrm{~mL} / \mathrm{L}$ FBS, $100 \mathrm{U} / \mathrm{mL}$ penicillin, and $100 \mu \mathrm{g} /$ $\mathrm{mL}$ streptomycin plus $1 / 10,2 / 10$, or $3 / 10 \mathrm{PRF}$ ) and three different adipogenic induction media (containing nutrient solution, $200 \mu \mathrm{M}$ indomethacin, $10 \mu \mathrm{M}$ insulin, $0.5 \mathrm{mM}$ 3 -isobutyl-1-methylxanthine, and $1 \mu \mathrm{M}$ dexamethasone plus $1 / 10,2 / 10$, or $3 / 10 \mathrm{PRF}$ ). The media were then stored at $4{ }^{\circ} \mathrm{C}$ until they were used in the following experiments.

We detected the slow release of growth factors, and the PRF membrane sample was transferred to a sterile tube with $4 \mathrm{~mL}$ of DMEM for preservation and incubated for 1, 24, or $72 \mathrm{~h}$. All DMEM samples were then stored at $-20^{\circ} \mathrm{C}$ until enzyme-linked immunosorbent assay (ELISA) quantification was performed. Vascular endothelial growth factor (VEGF), platelet derived growth factor-AB (PDGF-AB), transforming growth factor- $\beta$ (TGF- $\beta$ ), epidermal growth factor (EGF), interleukin-6 (IL-6), insulin-like growth factor (IGF-1), and matrix metallo-proteinase-1 (MMP-1) levels in the experimental PRF-conditioned DMEM samples were analyzed according to the ELISA kit manufacturer's protocol(MultiSciences, Zhejiang, CHN).

\section{Collection of nanofat}

Fat was collected using the Coleman method with some modifications and involved selecting a low area supply from the medial and lateral thigh, low negative pressure suction (20-mL reserved air method), and lowspeed centrifugation (1000 rpm/min for $2 \mathrm{~min}$ ). Depending on each patient's condition, local tumescent infiltration anesthesia was administered to the area of fat supply.

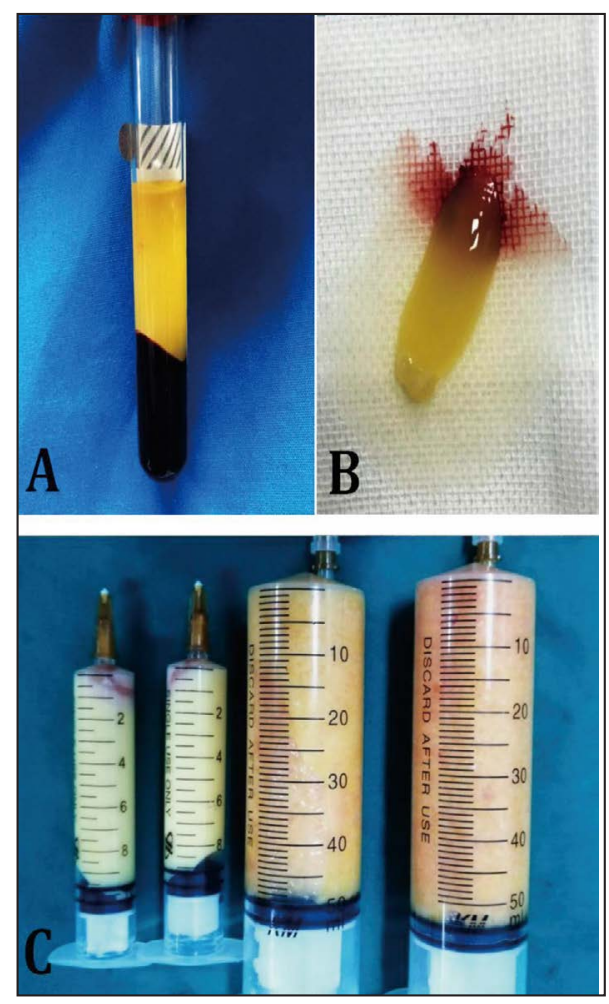

Fig. 1. A-B: Isolation of PRF clots from venous whole blood via centrifugation. After a single centrifugation, red blood cell elements were located in the lower phase, the middle phase containing PRF clots was transferred into a new tube to obtain the PRF precipitation liquid, and the upper phase consisted of platelet-poor plasma (PPP); C: Lipoaspirate was mechanically emulsified after rinsing. Nanofat in 10-mL syringes, structural fat in 50-mL syringes. 


\section{Cellular Physiology Cell Physiol Biochem 2018;47:316-329

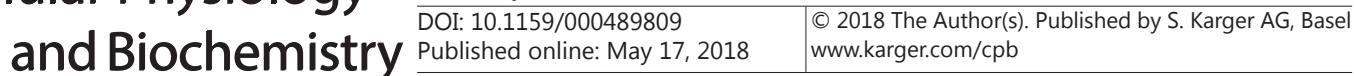 \\ Li et al.:Skin Rejuvenation Function of Nanofat-Derived Stromal Cells and Platelet-Rich}

Fibrin

Liposuction was performed using a 3.5-mm polyporous needle starting in the deep tissue and moving superficially repeatedly in a fan-shaped motion. The collected fat granules were prepared by washing with physiological saline (Fig. 1C). Nanofat was prepared by briefly mechanically emulsifying the rinsed lipoaspirate. Emulsification was achieved by transferring the fat between two 20-mL syringes connected to each other by a female-to-female Luer-Lok connector. After 3 min of continuous transfer, the fat became an emulsified liquid with a whitish appearance. The emulsified fat was filtered through a superfine filter to obtain nanofat (Fig. 1C).

\section{Isolated culture and identification of NFSCS}

The nanofat was then centrifuged at $8000 \mathrm{rpm}$ for $3 \mathrm{~min}$ and immediately resuspended without collagenase digestion. The enriched stromal vascular fraction (SVF) cell mass was resuspended with $10 \%$ fetal bovine serum (FBS) in DMEM nutrient solution. The resuspended cells were cultured in a dish in a $37^{\circ} \mathrm{C}$ incubator with $5 \% \mathrm{CO}_{2}$ to allow the NFSCs to proliferate. The nutrient solution in the dish was changed once every $24 \mathrm{~h}$, and cells that had not attached to the dish were removed. After that, the nutrient solution was changed once every 3 days. NFSC proliferation was monitored under a microscope. Once third-passage (P3) cells reached $80 \%$ to $90 \%$ confluence, they were resuspended in phosphate-buffered saline (PBS) with 30 $\mathrm{mL} / \mathrm{L}$ FBS, counted, and transferred into nine 1.5-mL centrifuge tubes. After they were trypsinized using Accutase (Innovative Cell Technologies, San Diego, CA), the P3 NFSCs were analyzed using flow cytometry after one of the following rabbit anti-human primary antibodies was added: CD29-PE, CD44-PE, CD49d-PE, CD73-PE, CD90-PE, or CD105-PE. Incubation with CD34-PE, CD45-PE, CD106-PE, or negative control IgG-PE antibodies was performed in the dark at room temperature for $30 \mathrm{~min}$. The cells were then washed with PBS three times, resuspended in PBS with $30 \mathrm{~mL} / \mathrm{L}$ FBS, and subjected to flow cytometry to measure the surface markers on NFSCs.

\section{Multipotency identification of NFSCs}

P3 NFSCs were cultured with adipogenic, osteogenic, and chondrogenic induction media, and lineagespecific cell morphologies were observed after 2 or 3 weeks. Adipocytes, osteoblasts, and chondrocytes were identified by positive Oil Red 0, alizarin red, and Alcian blue staining, respectively. Details of the experimental methods are described in our previous reports $[10,11]$. Cells in all induction media were cultured for 14 days. Total RNA was extracted from each group using TRIzol according to the manufacturer's protocol (Invitrogen, USA). First-strand cDNA was synthesized from $1 \mu \mathrm{g}$ of RNA with viral reverse transcriptase (TaKaRa, Japan) and used for real-time qPCR. PPAR $\gamma$, ALP and COL-II mRNA expression were measured(PPAR $\gamma$ : forward, AATCAAAGTGGAACCTGCATC, reverse, TTCGGAAGAAACCCTTGCAT, annealing temperature, $57^{\circ} \mathrm{C}$; ALP: $^{\circ}$ forward,TGGAGCTTCAGAAGCTCAACACCA,reverse,ATCTCGTTGTCTGAGTACCAGTCC, annealingtemperature, $58^{\circ} \mathrm{C}$; COL-II: forward, CTATCTGGACGAAGCAGCTGGCA, reverse, ATGGGTGCAATGTCAATGATGG, annealing temperature, $58^{\circ} \mathrm{C}$; GAPDH: forward, ATGTTGTCGCCATCAATGATCC, reverse, GTACTCGGCACCAGCATCAC, annealing temperature, $58^{\circ} \mathrm{C}$;). This experiment was performed in triplicate.

\section{Measurement of PRF-induced proliferation and paracrine function in NFSCS}

P3 human NFSCs were examined using the Cell Counting Kit-8 (CCK-8) method. The cells were transferred to a 96 -well plate $\left(1 \times 10^{4}\right.$ cells/well) and cultured for $24 \mathrm{~h}$ at $37^{\circ} \mathrm{C}$ with $50 \mathrm{~mL} / \mathrm{L} \mathrm{CO}_{2}$, after which the nutrient solution was poured off. Cells were then randomly divided into 4 groups with three wells per group ( $n=3$ ); for the three experimental groups (groups 2,3 , and 4 ), culture medium with 1/10, $2 / 10$, or 3/10 PRF, respectively, was added, while common culture medium was added for the control group (group 1). After 9 additional days, during which the supernatant was removed daily, $50 \mu \mathrm{L}$ of CCK-8 working solution was added to each well after culture for $4 \mathrm{~h}$. After a 10-min incubation, the optical density (OD) at $450 \mathrm{~nm}$ wavelength was detected using a microplate reader. Real-time qPCR and western blotting (WB) (Abcam, London, UK) were performed according to the manufacturer's instructions, and the mRNA and protein expression of VEGF, b-FGF, PDGF, and TGF- $\beta$ were measured after co-culture for $72 \mathrm{~h}$ (VEGF: forward, CTTGCCTTGCTGCTCTACCTCC, reverse, CACACTCCAGGCCCTCGTCA, annealing temperature, $63^{\circ} \mathrm{C}$; b-FGF: forward, GCGACCCTCACATCAAGCTAC, reverse, CCAGTTCGTTTCAGTGCCACA, annealing temperature, $60^{\circ} \mathrm{C}$; PDGF: forward, CCACTAAGCATGTGCCCGAGA, reverse, TGGCACTTGACACTGCTCGT, annealing temperature, $58^{\circ} \mathrm{C}$; TGF- $\beta$ : forward, TACCTGAACCCGTGTTGCTCT, reverse, CTGCCGCACAACTCCGGTGA, annealing temperature, $62^{\circ} \mathrm{C}$ ). This experiment was repeated in triplicate. 


\section{Cellular Physiology Cell Physiol Biochem 2018;47:316-329

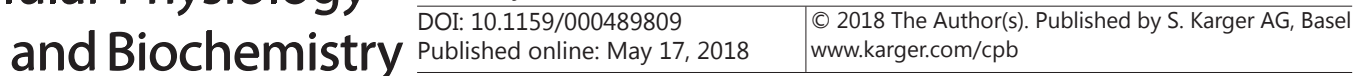 Li et al.:Skin Rejuvenation Function of Nanofat-Derived Stromal Cells and Platelet-Rich Fibrin}

\section{Scanning electron microscopy (SEM)}

After NFSCs and PRF membranes were co-cultured for $72 \mathrm{~h}$, the morphology of cells growing on the PRF membrane was evaluated using SEM (PhenomProX; Phenom-World BV, Eindhoven, The Netherlands) with images taken on an SEM-FE MIRA II LMU (TESCAN, Brno-Kohoutovice, Czech Republic). Samples were dehydrated and fixed in 2.5\% glutaraldehyde; washed in PBS; dehydrated in 50, 70, 80, 95, and 100\% EtOH mixed with hexamethyldisilazane (HMDS) (Sigma, Aldrich) and 100\% HMDS solution; and then were coated with gold/palladium. ImageJ software was used for image analysis.

\section{Intradermal injection of the NFSC-PRF mixture or HA}

Nanofat and PRF were mixed at the proper proportions for intradermal injection. Facial skin surface anesthesia was provided by applying lidocaine ointment. The NFSC-PRF mixture or HA was injected into the dermis of the forehead, cheeks, and chin area in nine locations with needle hydro lifting, with a total volume of 4-5 mL for each patient and a stable positive pressure. After injection, a frozen sterile HA mask was immediately applied to help stop bleeding and relieve discomfort.

\section{Follow-up}

Photographs of patients' faces were taken at various time points pre- and post-injection and were used to evaluate skin quality. Follow-up was done at 1, 6, 12, and 24 months after injection for all 103 treatment patients and 128 controls. All photos used for evaluation were taken by the same doctor with the same camera and illumination source and from the same angle. The VISIA Skin Image Analyzer and a SOFT5.5 skin test instrument were used for quantitative measurements. Examinations were conducted at $24^{\circ} \mathrm{C}$ and $40-50 \%$ humidity. The SOFT5.5 skin tester was placed vertically on the skin, and pH, moisture, elasticity, and sebum level were measured after the reading had stabilized for 5-10 s. These measurements were repeated 5 times and averaged. The VISIA Skin Image Analyzer was used to photograph the subjects' faces while minimizing the effects of reflected light and obstruction by hair as much as possible. The VISIA Skin Image Analyzer automatically generated an absolute score that indicated the strength of the skin in the chosen area for each patient.

\section{Observation index and curative effect}

The observation index accounted for differences in facial skin texture before and after treatment, complications that occurred, and degree of satisfaction for all patients. Curative effect was defined as follows:Excellent (very satisfied): firm skin and obvious improvement in signs of aging after treatment; Good (satisfied): obvious improvement in signs of aging; Average (partially satisfied): some improvement in signs of aging after treatment; Poor (unsatisfied): minimal changes in facial appearance after treatment.

\section{Statistical analysis}

Data are expressed as the mean \pm standard deviation; statistical analyses were performed using SPSS17.0 software. Multi-group measurements were compared using one-way analysis of variance, and comparisons between two groups were evaluated using the Scheffe post hoc test. Enumerated data were analyzed using the $\chi^{2}$ test. A P value $<0.05$ indicated statistical significance.

\section{Results}

\section{Growth factor concentrations in $P R F$}

Levels of VEGF, b-FGF, EGF, PDGF-AB, IGF-1, TGF- 31 , IL-4, IL-6, and MMP-1 secreted by the PRF increased gradually in a time-dependent manner after isolation in culture, and peaks appeared on day 7. Growth factor levels at different timepoints are shown in Fig. 2.

\section{Biological characteristics of NFSCs}

After initial isolation and culture, most primary NFSCs became spindle shaped and adherent to the dish, forming a monolayer in 24 to $48 \mathrm{~h}$, while a few triangular or polygonal cells were also observed (Fig. 3A). With continued culture, NFSCs grew as more typical fusiform cells with colonies of different sizes (Fig. 3B). P1 NFSCs reached 80-90\% confluence 10 to 12 days after initial seeding (Fig. 3C) and were passaged at a ratio of 1:3. The passaged cells grew markedly faster and reached the same confluence within 3 days compared with 
primary cells. All NFSC passages shared similar shapes and growth patterns with ASCs, which were obtained via the conventional collagenase digestion method described in our previous study [6]. After 2 weeks of inductive culture for adipocytes, osteoblasts, and chondrocytes, positive staining for Oil Red 0, alizarin red, and Alcian blue in cells indicated the multipotency of the NFSCs (Fig. 3E, G, I). Immunofluorescence staining demonstrated positive expression of CD29, CD44, CD49d, CD73, CD90, and CD105, while no expression of CD34, CD45, and CD106 was observed in P3 NFSCs (Fig. 4).

\section{Effects of PRF on NFSC} proliferation

NFSCs were cultured in media containing PRF concentrations of $0 \%, 10 \%, 20 \%$, or $30 \%$, and CCK- 8 tests were performed on these 4 groups at 1 to 9 days. OD values of all experimental groups (including groups 2,3 , and 4 of NFSCs respectively treated with PRF concentrations of $1 / 10,2 / 10$, or $3 / 10$ ) were higher than those of the control group (group 1, only NFSCs) at all timepoints starting from the third day $\left({ }^{*} \mathrm{P}<\right.$ 0.05 ), and cellular growth of all groups reached a plateau at day 6. Growth curves clearly showed that PRF affected NFSC proliferation in a doseand time-dependent manner (Fig. 5E). After co-culture for 3 days, NFSCs began migrat-

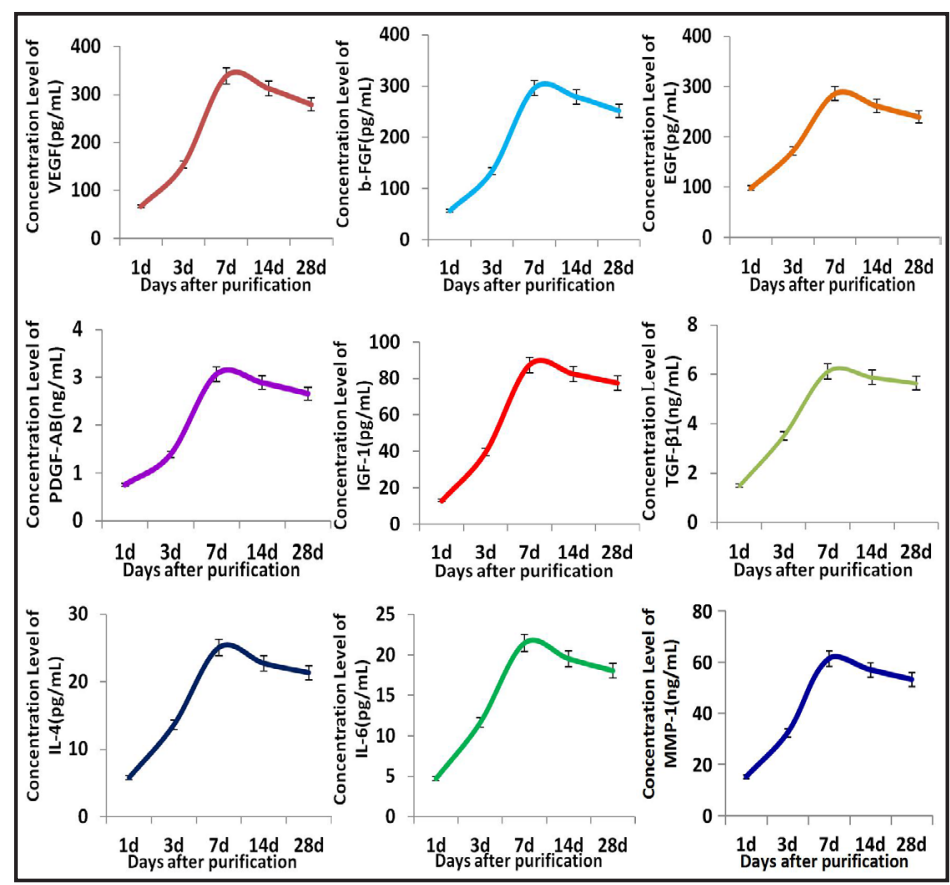

Fig. 2. Levels of VEGF, b-FGF, EGF, PDGF-A $\beta$, TGF- $\beta 1$, IL-4, IL-6, IGF-1, and MMP-1 secreted by the PRF increased gradually in a time-dependent manner.

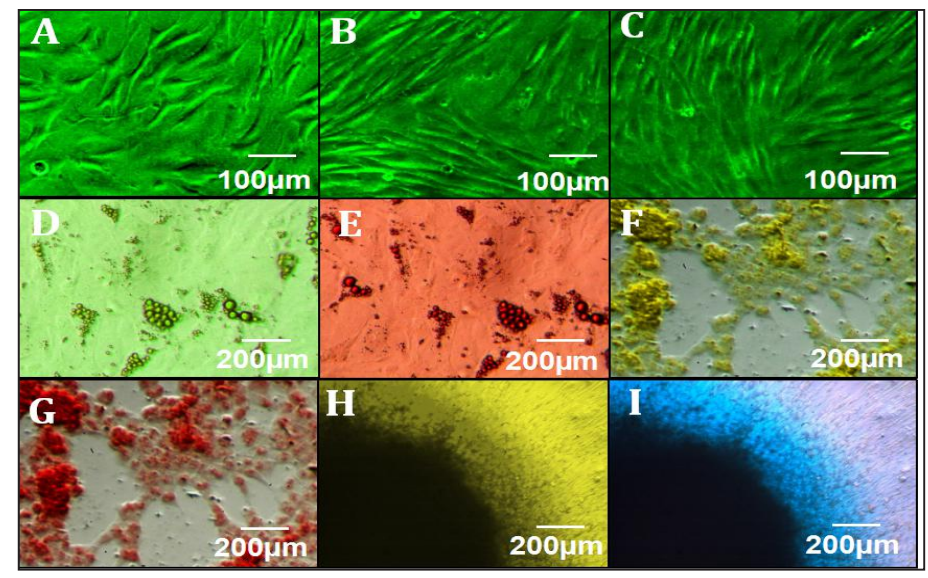

Fig. 3. Characterization of NFSCs. A: Initial isolation and culture of primary NFSCs for $24 \mathrm{~h}$ to $48 \mathrm{~h}$. B: NFSCs cultured more than $48 \mathrm{~h}$. C: Morphological characterization of primary NFSCs. D: Adipogenic induction for 2 weeks before staining. E: Positive Oil Red 0 staining after 2 weeks of induction culture for adipocytes. F: Osteogenic induction for 2 weeks before staining. G: Alizarin red staining of induction culture for osteoblasts after 2 weeks. H: Chondrogenic induction for 3 weeks before staining. I: Alcian blue staining after 2 weeks of induction culture for chondrocytes. ing into the pores of the PRF membrane. SEM revealed cells extending pseudopodia and adhering to the well surface or pores, aggregating, and growing gradually on the scaffold; nascent extracellular matrix was also observed (Fig. 5A-D). 
Fig. 4. Immunofluorescence staining of P3 NFSCs demonstrating positive expression of CD29, CD44, CD49d, CD73, CD90, and CD105, and negative expression of CD34, CD45, and CD106(CD29: 93.4 \pm 0.8 ,

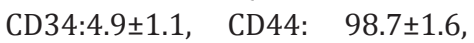
CD45: $3.6 \pm 0.9$, CD49: $88.5 \pm 3.3$, CD73: 90.9 \pm 2.8, CD90: 92.8 \pm 4.1 , CD105: 97.5 \pm 1.9, CD106:3.1 \pm 0.7 ; Mean $\pm S D, n=6$ ).

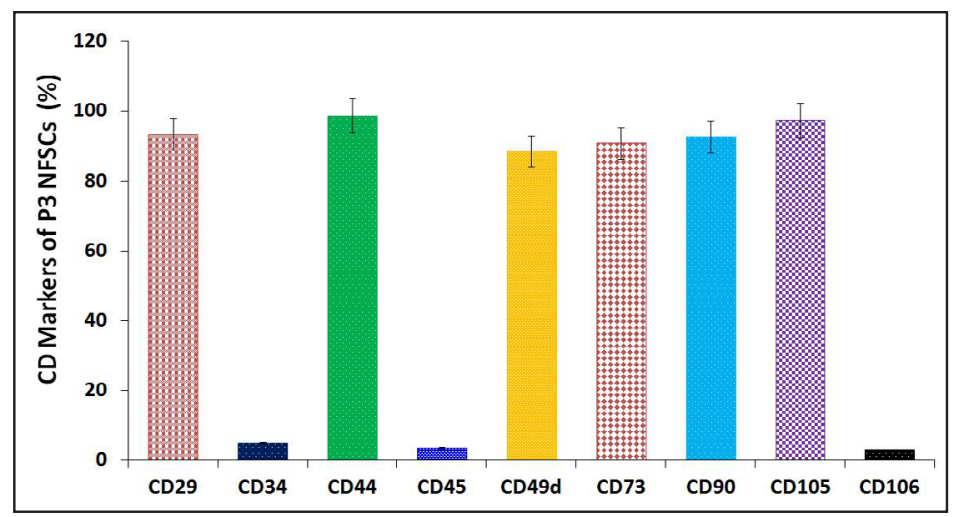

Fig. 5. Morphology and status of NFSCs after co-culture with PRF. A: Morphological characterization of NFSCs under RLM after co-culture with PRF for $24 \mathrm{~h}$. B: Morphological characterization under RLM of NFSCs after co-culture with PRF for $72 \mathrm{~h}$. C: NFSCs under SEM after 72 h of co-culture. SEM: $\times 20.0 \mathrm{k}$. D: NFSCs under SEM after 7d of co-culture. E: NFSCs were cultured in medium without PRF (group 1) or cultured in media containing PRF concentrations of $1 / 10,2 / 10$, $3 / 10$ (groups 2-4). CCK-8 tests were performed on these 4 groups at 1 to 9 days. The results are shown as the mean; $\mathrm{P}<0.05$.

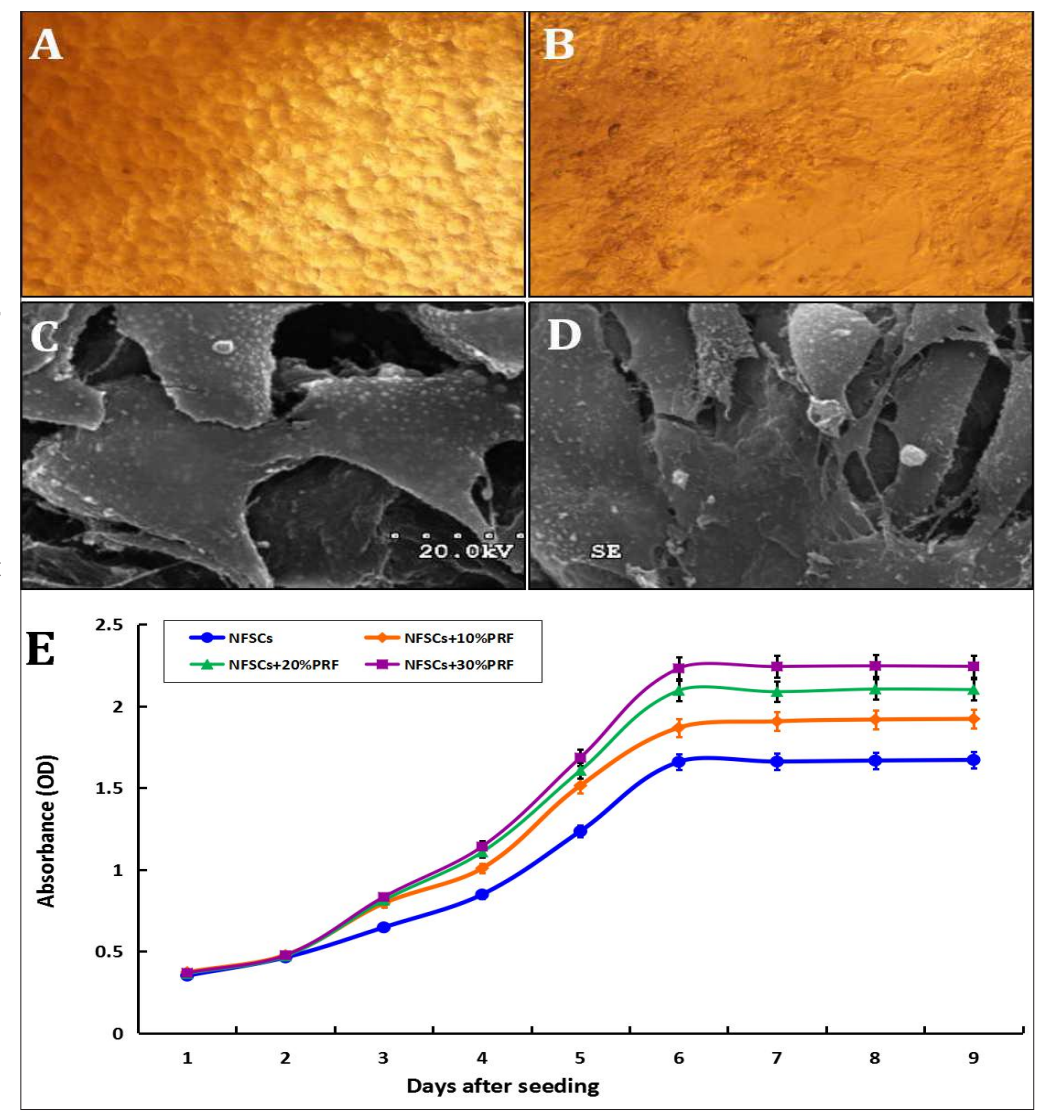

\section{Influences of PRF on NFSC multipotency}

P3 NFSCs were cultured in 6-well culture plates and then distributed into two groups: experimental groups treated with different orientation induction media (adipogenic, osteogenic, and chondrogenic) plus 1/10 PRF, whereas the control groups were treated with orientation induction medium alone. After 14 days of induction, 6 samples were harvested from each group for real-time PCR analysis. Relative mRNA expression of PPAR $\gamma$, ALP, and $\mathrm{CO}-\mathrm{II}$ in the experimental groups was higher than that in the control group $\left({ }^{*} \mathrm{P}<0.05\right.$, Fig. 6 ).

\section{Effects of PRF on NFSC paracrine function}

At day 6 after culture, the paracrine function of NFSCs was evaluated by determining relative mRNA expression and protein level of several growth factors using real-time qPCR assay and WB. Compared with the expression seen in the control group (NFSCs only), the mRNA expression and protein level of VEGF, b-FGF, PDGF, and TGF- $\beta$ was much higher in NFSCs cultured with $1 / 10$ PRF (Fig. $7,{ }^{*} \mathrm{P}<0.01$ ). 


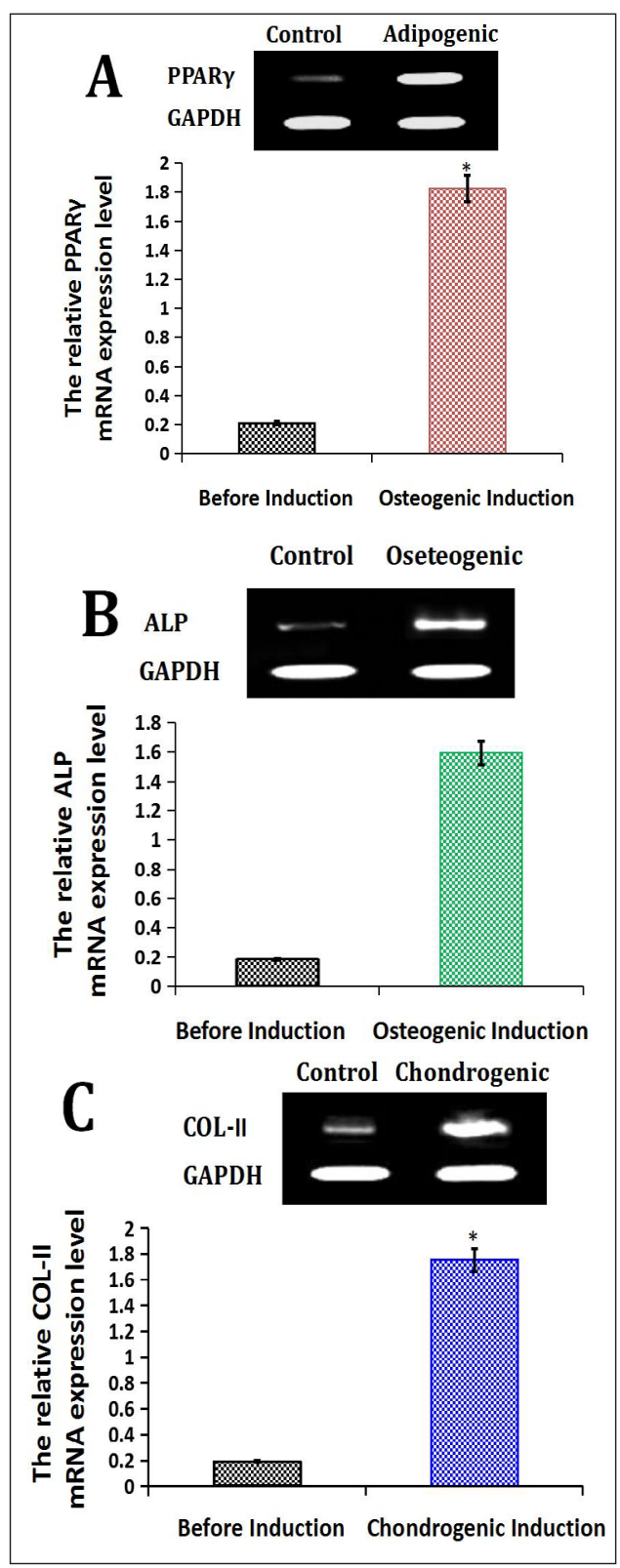

Fig. 6. Comparison of the relative mRNA expression of NFSCs in groups cultured without and with multiple differentiation induction medium (A: adipogenic medium; B: osteogenic medium; $\mathrm{C}$ : chondrogenic medium, ${ }^{*} \mathrm{P}<0.05$ ).
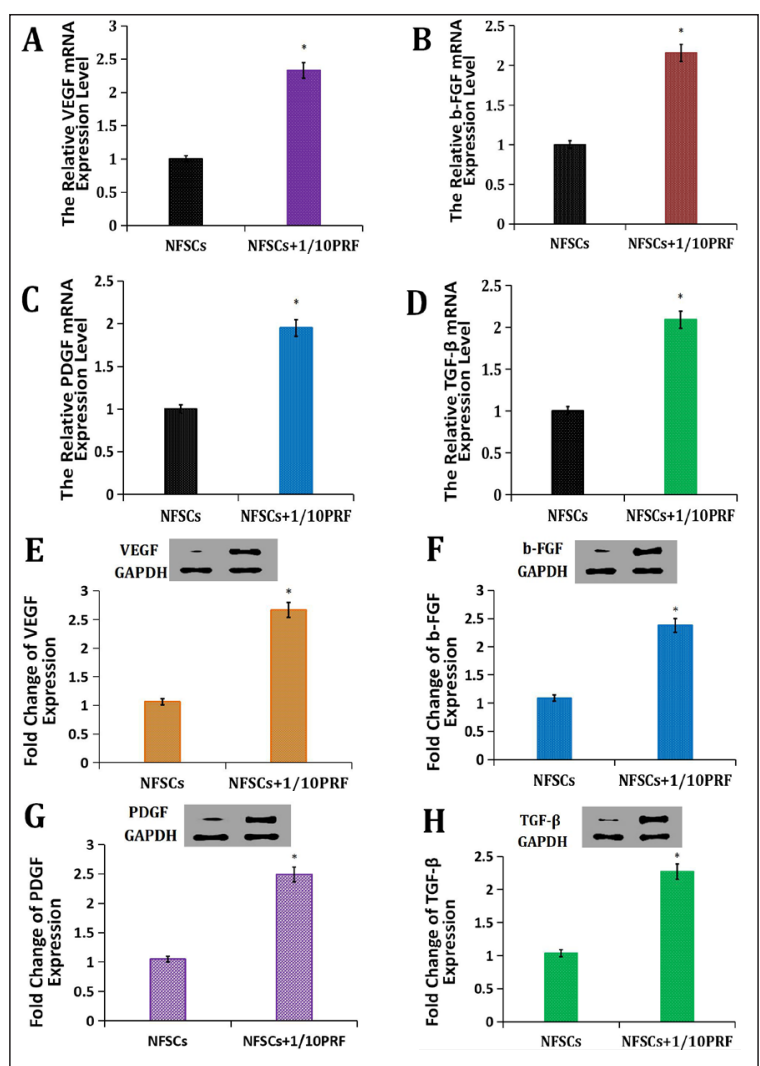

Fig. 7. A-D: Relative mRNA (VEGF, b-FGF, PDGF, TGF- $\beta$ ) expression of cells in the experimental (NFSCs plus $1 / 10 \mathrm{PRF})$ and control groups (NFSCs only) $\left({ }^{*} \mathrm{P}<0.05\right)$. E-H: Western blot (WB) analysis of VEGF, b-FGF, PDGF, and TGF- $\beta$ protein level in the experimental (NFSCs plus $1 / 10 \mathrm{PRF})$ and control groups (NFSCs only) $\left({ }^{*} \mathrm{P}<0.05\right)$.

\section{Analysis of clinical efficacy}

A statistically significant difference in general skin appearance before and at 6 months after treatment was achieved in all patients. Patients, plastic surgeons, and independent third parties independently assessed skin condition using pre- and postoperative photos to evaluate the effects of the injections. Satisfaction rates were higher in the test group than in the control group after 6 and 12 months (Table 1).

No significant or persistent complications were observed in any patients during followup. Transient infection occurred in 5 patients and paresthesia occurred in 7 patients within 
Table 1. Three methods for evaluating satisfaction at 6 and 12 months in the comprehensive treatment and control groups (cases, \%) - A, Patients; B, Surgeons; C, Independent third party I, Very Satisfied; II, Satisfied; III, Average; IV, Unsatisfied

\begin{tabular}{lccccc}
\hline Evaluators & $\mathrm{n}$ & $\mathrm{I}$ & $\mathrm{II}$ & $\mathrm{III}$ & IV \\
\hline & & & 6 months & & \\
$\mathrm{A}$ & $103 \mathrm{vs}$ & $84(81.55) \mathrm{vs}$ & $14(13.59) \mathrm{vs}$ & $5(4.86) \mathrm{vs}$ & $0(0) \mathrm{vs}$ \\
& 128 & $37(28.91)$ & $52(40.62)$ & $22(17.19)$ & $17(13.28)$ \\
$\mathrm{B}$ & $103 \mathrm{vs}$ & $85(82.52) \mathrm{vs}$ & $14(13.59) \mathrm{vs}$ & $4(3.89) \mathrm{vs}$ & $0(0) \mathrm{vs}$ \\
& 128 & $38(29.69)$ & $53(41.40)$ & $21(16.41)$ & $16(12.50)$ \\
$\mathrm{C}$ & $103 \mathrm{vs}$ & $80(77.67) \mathrm{vs}$ & $16(15.53) \mathrm{vs}$ & $7(6.80) \mathrm{vs}$ & $0(0) \mathrm{vs}$ \\
& 128 & $35(27.34)$ & $55(42.97)$ & $21(16.41)$ & $17(13.28)$ \\
& $103 \mathrm{vs}$ & $82(79.61) \mathrm{vs}$ & $12(11.65) \mathrm{vs}$ & $9(8.74) \mathrm{vs}$ & $0(0) \mathrm{vs}$ \\
$\mathrm{A}$ & 128 & $19(14.84)$ & $47(36.72)$ & $37(28.91)$ & $25(19.53)$ \\
& $103 \mathrm{vs}$ & $83(80.58) \mathrm{vs}$ & $13(12.62) \mathrm{vs}$ & $7(6.80) \mathrm{vs}$ & $0(0) \mathrm{vs}$ \\
$\mathrm{B}$ & 128 & $20(15.63)$ & $48(37.50)$ & $35(27.34)$ & $25(19.53)$ \\
& $103 \mathrm{vs}$ & $79(76.70) \mathrm{vs}$ & $15(14.56) \mathrm{vs}$ & $8(7.77) \mathrm{vs}$ & $1(0.97) \mathrm{vs}$ \\
$\mathrm{C}$ & 128 & $19(14.84)$ & $46(35.94)$ & $37(28.91)$ & $26(20.31)$ \\
\hline
\end{tabular}

Table 2. Incidence rates of postoperative complications in the comprehensive treatment group $(n=103)$ and the control group $(n=128)$ - Values are the number of cases $n(\%){ }^{*} P>0.05$ vs control group, ${ }^{\#} \mathrm{P}<0.05$ vs control group.

\begin{tabular}{lcccccc}
\hline $\begin{array}{l}\text { Stage (post- } \\
\text { operative) }\end{array}$ & Group & Infection & Pigmentation & Anaphylaxis & Topoparesthesia & Rate \\
\hline 7 days & Treatment & $2(1.94)$ & $3(2.91)$ & $0(0)$ & $9(8.74)$ & $13.59^{*}$ \\
& Control & $3(2.34)$ & $4(3.13)$ & $0(0)$ & $11(8.59)$ & 14.06 \\
6 months & Treatment & $0(0)$ & $1(9.71)$ & $0(0)$ & $0(0)$ & $9.71^{\#}$ \\
& Control & $0(0)$ & $1(9.71)$ & $0(0)$ & $0(0)$ & 7.82 \\
12 months & Treatment & $0(0)$ & $1(9.71)$ & $0(0)$ & $0(0)$ & $9.71^{\#}$ \\
& Control & $0(0)$ & $1(7.82)$ & $0(0)$ & $0(0)$ & 7.82 \\
\hline
\end{tabular}

Table 3. VISIA and SOFT5.5 values for facial skin in the comprehensive treatment group $(\mathrm{n}=103)$ and control group $(n=128)$ - All values expressed as the mean \pm standard deviation. ${ }^{*}$ Comparison within treatment group, $\mathrm{P}<0.01$ vs before; "Comparison within treatment group, $\mathrm{P}<0.01$ vs before

\begin{tabular}{|c|c|c|c|c|c|c|c|c|}
\hline & \multicolumn{2}{|c|}{ Before } & \multicolumn{2}{|c|}{1 Month } & \multicolumn{2}{|c|}{12 Months } & \multicolumn{2}{|c|}{24 Months } \\
\hline & $\begin{array}{c}\text { Treatment } \\
\text { group }\end{array}$ & $\begin{array}{l}\text { Control } \\
\text { group }\end{array}$ & $\begin{array}{l}\text { Treatment } \\
\text { group }\end{array}$ & $\begin{array}{l}\text { Control } \\
\text { group }\end{array}$ & $\begin{array}{l}\text { Treatment } \\
\text { group }\end{array}$ & $\begin{array}{l}\text { Control } \\
\text { group }\end{array}$ & $\begin{array}{l}\text { Treatment } \\
\text { group }\end{array}$ & $\begin{array}{l}\text { Control } \\
\text { group }\end{array}$ \\
\hline \multicolumn{9}{|l|}{ VISIA values } \\
\hline Splash & $51.04 \pm 9.38$ & $52.03 \pm 10.47$ & $44.11 \pm 9.05^{*}$ & $43.85 \pm 9.76^{\#}$ & $41.24 \pm 8.73^{*}$ & $44.59 \pm 9.68^{\#}$ & $41.96 \pm 9.13^{*}$ & $51.88 \pm 10.25$ \\
\hline Wrinkles & $59.17 \pm 11.43$ & $58.73 \pm 11.26$ & $35.65 \pm 10.69^{*}$ & $39.19 \pm 9.62 \#$ & $33.53 \pm 10.28^{*}$ & $57.84 \pm 11.07$ & $33.89 \pm 10.17^{*}$ & $58.26 \pm 10.78$ \\
\hline Texture & $53.78 \pm 12.06$ & $54.65 \pm 10.97$ & $41.08 \pm 11.45^{*}$ & $45.24 \pm 10.06^{\#}$ & $39.76 \pm 11.01^{*}$ & $53.89 \pm 10.77$ & $38.75 \pm 10.93^{*}$ & $55.02 \pm 11.14$ \\
\hline Pore & $64.94 \pm 14.25$ & $66.26 \pm 13.49$ & $55.47 \pm 13.29^{*}$ & $61.07 \pm 13.15^{\#}$ & $53.84 \pm 13.13^{*}$ & $62.27 \pm 12.95^{\#}$ & $56.07 \pm 12.92^{*}$ & $65.99 \pm 14.18$ \\
\hline Sclererythrin & $36.88 \pm 7.85$ & $48.25 \pm 9.57$ & $27.42 \pm 5.63^{*}$ & $32.21 \pm 7.65^{\#}$ & $29.49 \pm 5.98^{*}$ & $47.89 \pm 8.42$ & $30.15 \pm 6.048^{*}$ & $46.88 \pm 9.12$ \\
\hline \multicolumn{9}{|l|}{$\begin{array}{l}\text { SOFT5.5 } \\
\text { values }\end{array}$} \\
\hline $\mathrm{pH}$ & $5.63 \pm 0.72$ & $5.93 \pm 0.89$ & $5.49 \pm 0.68$ & $5.82 \pm 0.77$ & $5.38 \pm 0.71$ & 5.78 & $5.57 \pm 0.74$ & $5.86 \pm 0.84$ \\
\hline Moisture & $49.05 \pm 7.21$ & $51.28 \pm 7.91$ & $75.24 \pm 9.23^{*}$ & $69.52 \pm 9.43^{\#}$ & $71.96 \pm 9.04^{*}$ & $50.76 \pm 8.05$ & $67.85 \pm 8.83^{*}$ & $51.66 \pm 8.04$ \\
\hline Elasticity & $12.47 \pm 3.69$ & $11.02 \pm 3.26$ & $16.86 \pm 4.12^{*}$ & $15.17 \pm 4.23^{\#}$ & $15.94 \pm 4.82^{*}$ & $10.89 \pm 3.43$ & $14.87 \pm 4.64^{*}$ & $11.15 \pm 3.47$ \\
\hline Grease & $47.25 \pm 10.28$ & $49.22 \pm 9.14$ & $33.42 \pm 9.86^{*}$ & $35.29 \pm 8.75^{\#}$ & $37.65 \pm 10.03^{*}$ & $47.96 \pm 8.83$ & $38.92 \pm 10.25^{*}$ & $48.77 \pm 9.09$ \\
\hline
\end{tabular}

7 days after injection who completely recover later, while 2 patients $(1$ each from the test group and the control group) reported pigmentation changes that lasted for more than 12 months. No patients experienced anaphylaxis or an allergic reaction (Table 2). Scores for improvements in skin splash, wrinkles, texture, pores, sclererythrin test, $\mathrm{pH}$, moisture, elasticity, and sebum based on VISIA and SOFT5.5 analysis are shown in Table 3. There was a statistically significant improvement in skin condition in both groups, but improvements were sustained for more than 12 months in the test group compared with 1 month in the control group (Table 3). 


\section{Cellular Physiology Cell Physiol Biochem 2018;47:316-329 \\ and Biochemistry Published online: May 17, $2018 \quad \begin{aligned} & \text { DOI: 10.1159/000489809 } 2018 \text { The Author(s). Published by S. Karger AG, Basel } \\ & \text { www.karger.com/cpb }\end{aligned}$ \\ Li et al.:Skin Rejuvenation Function of Nanofat-Derived Stromal Cells and Platelet-Rich \\ Fibrin}

\section{Discussion}

In recent years, fat grafts have been widely used in the treatment of the aging of facial skin to fill in surface depressions [5, 12-14]. Many clinicians have noted that implanted fat tissue produces rejuvenation effects on skin surrounding the transplantation area $[1,12$, 13]. However, the biological mechanism responsible for the rejuvenating and regenerative effects of fat transplantation on skin are not yet fully understood [2]. Several studies have been conducted to assess the efficacy of fat grafting on facial rejuvenation [1, 15-17], with the results suggesting that such rejuvenation effects result from the improvement of deep facial structures. In contrast, others have suggested that ASCs, which are abundant in fat tissue, participate in soft tissue remodeling by promoting collagen synthesis, cellular matrix protein synthesis, and revascularization of the dermis via the production of many cytokines and multipotential proliferation [17-20].

Human adipose tissue is composed of $90 \%$ mature adipocytes and a SVF that includes abundant ASCs. ASCs have outstanding self-renewal potential, paracrine function, and the capacity to differentiate into multiple phenotypes. Some data have suggested the application of ASCs in lesions induces healing because of the role of ASCs in stimulating angiogenesis, which is attributed to their endothelial differentiation and release of several cytokines under specific conditions [21-24].

To date, SVF grafting only, without mature adipocytes in the adipose tissue component, has been widely used in cosmetic surgery $[25,26]$, while the conventional approach involves ASC injection alone. In clinical practice, the procedure for isolating ASCs from adipose tissue primarily involves collagenase digestion, which is heavily regulated through good manufacturing practice guidelines regulatory rules [27]. To simplify the processing procedure, some researchers have introduced a technique where SVF is injected into the subcutaneous layer only, without mature adipocytes, and reported good anti-aging effects on skin $[28,29]$.

Nanofat is an emulsified suspension rich in SVF, which was proposed for use in antiaging medicine for the first time by Tonnard et al. in 2013 [4]. The use of nanofat has been generalized and applied for cosmetic treatment in recent years [30,31]. In the present study, NFSCs were isolated successfully and then identified by induction of multipotent differentiation and flow cytometry. The NFSCs possessed excellent multilineage potential (Fig. 3D-I) and had the same surface CD-antigen expression as ASCs harvested from structural fat, as shown in our previous study (Fig. 4) [11, 32]. Since nanofat is virtually devoid of mature adipocytes, its regenerative functions are primarily ascribed to NFSCs, and several studies have been performed to optimize the preparation process to obtain higher NFSC density [33].

In addition to the number of cells, the biological function of NFSCs also affects their regenerative efficacy. Based on our previous studies, PRF is an excellent adjuvant for promoting the proliferation, differentiation, and paracrine function of ASCs [6]. In addition, functional optimization of NFSCs produced by PRF was also observed in this study (Figs. 6 and 7). PRF has been widely used in clinical practice to improve the efficacy of cell-assisted lipotransfer (CAL) and wound repair by ASCs, due to its advantages in preventing immunologic rejection, simple production, and few complications. Moreover, plastic surgeons have adopted PRP, a predecessor of PRF, as a rejuvenator to treat aging skin by intradermal injection [8, 34, 35].

Large amounts of growth factors and immunologic factors released by PRF are secreted from the $\alpha$-granules of concentrated platelets, which are crucial for the properties of PRF to accelerate the repair of aged and damaged soft tissues [36, 37]. In addition, PRF not only represents a rich source of growth factors but also provides a scaffold to support true tissue regeneration. Our results indicated that the concentrations of cytokines-including VEGF, b-FGF, EGF, PDGF-A $\beta$, TGF- $\beta 1$, IL-4, IL-6, IGF-1, and MMP-1-released from PRF gradually increased after purification and peaked on day 7 of culture (Fig. 2). SEM observation of cocultured NFSCs with PRF clots revealed that NFSCs began migrating into the pores, extended 


\section{Cellular Physiology Cell Physiol Biochem 2018;47:316-329

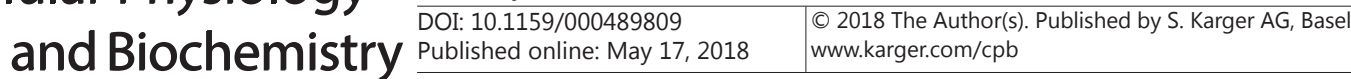 \\ Li et al::Skin Rejuvenation Function of Nanofat-Derived Stromal Cells and Platelet-Rich Fibrin}

pseudopodia, adhered to the well surfaces, aggregated, and gradually grew on the scaffold where they were adequately stimulated by cytokines.

Skin hydration is affected by HA concentration, which is involved in maintaining the skin framework. As a result of the ability of HA to form hydrated, expanded matrices, HA has also been successfully used for facial skin rejuvenation via intradermal-injection [38, 39]. However, the micromolecular structure of HA shortens the duration of its efficacy. Since the anti-aging efficacy produced by a single application of nanofat or PRF is significant [4, 37], we proposed a technique to precisely inject an intradermal nanofat-PRF mixture using nine sites for better rejuvenation (Fig. 8A). Most enrolled patients in our study reported notable satisfaction 6 months and 12 months after treatment, and patients in the comprehensive treatment group receiving injections of nanofatPRF had a higher and more durable mean satisfaction rate than the control group treated with HA (Table 1).

In addition to the subjective evaluation, the scores of facial skin condition from VISIA and SOFT5.5 tests showed that patients in each group achieved significant skin quality enhancement at 1 month $(\mathrm{P}<0.01$ vs. before treatment). These scores at 12 months were still significantly different from the pre-treatment scores in the comprehensive treatment group

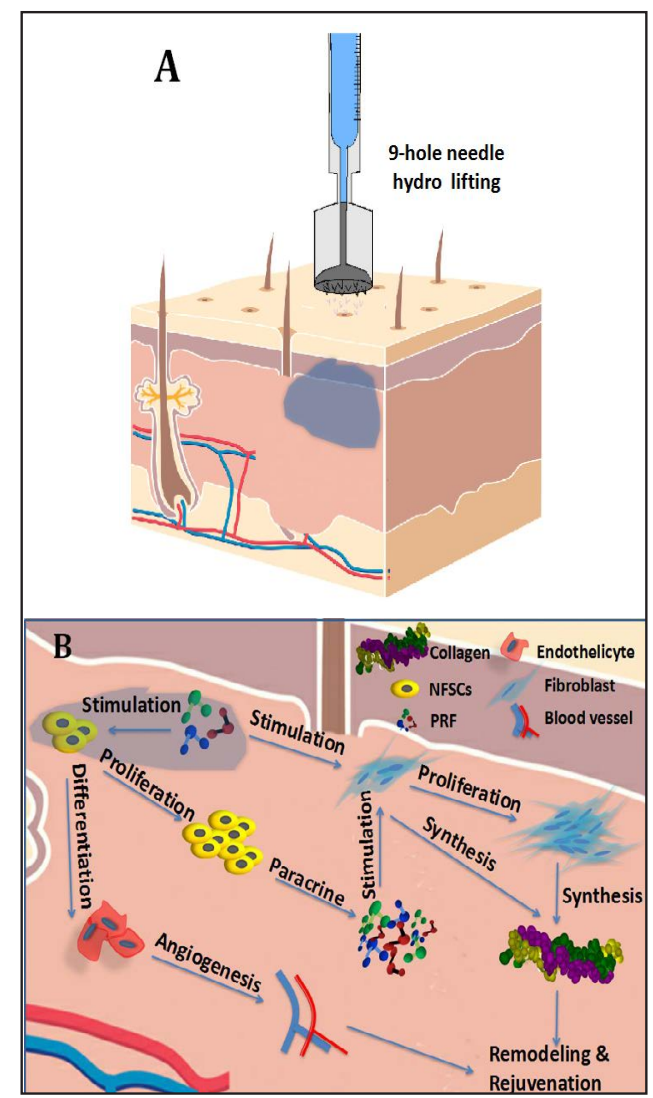

Fig. 8. A: Precise intradermal injection of nanofat-PRF mixture or HA with 9-hole microneedle hydro lifting. B: Mechanism by which nanofat-PRF mixture promotes skin regeneration and dermal rejuvenation. but not in the control group (Table 3).The results revealed that nanofat-PRF produced a more durable rejuvenation benefit and may be a more promising biological agent.

According to the in vitro experimental results, PRF acted as a main source of cytokines during early stages before NFSC adherence and simultaneously promoted the proliferation and paracrine function of NFSCs. These are the key roles of PRF in tissue regeneration and repair. We showed that nanofat-PRF in the dermis also functions in this mode, inducing synergistic and long-lasting effects. PRF consistently released growth factors, stimulating proliferation and paracrine function of NFSCs, and leading to a significant increase in growth factor concentration in the dermis, thus promoting fibroblasts proliferation and collagen generation. In addition, the enriched growth factors promote differentiation of stem cells into vascular endothelial cells, enhance in situ tissue angiogenesis, and provide more nutrients to create favorable conditions for repair and regeneration of skin structures (Fig. 8B). Furthermore, the low incidence of complications demonstrated that the injection of nanofat$\mathrm{PRF}$ combination is an effective and safe technique for facial skin rejuvenation (Table 2).

\section{Conclusion}

This study showed that ASCs isolated from nanofat possessed excellent proliferation, multilineage differentiation, and paracrine function, and the biological functions of the ASCs subset of NFSCs were significantly improved by PRF. The clinical outcomes demonstrated 


\section{Cellular Physiology Cell Physiol Biochem 2018;47:316-329 and Biochemistry Published $\begin{aligned} & \text { DOI: 10.1159/000489809 } \\ & \text { (c) } 2018 \text { The Author(s). Published by S. Karger AG, Basel } \\ & \text { www.karger.com/cpb }\end{aligned}$ \\ Li et al.:Skin Rejuvenation Function of Nanofat-Derived Stromal Cells and Platelet-Rich Fibrin}

that precise intradermal injection of nanofat-PRF was safe and highly effective for facial rejuvenation and repair.

\section{Acknowledgements}

This work was financially supported by the National Natural Science Foundation of China (81560316, 81760346), the Scientific Research and Technology Development Program of Guangxi (Guikegong 1598012-1), the Guangxi Natural Science Foundation (2016GXNSFDA380016), the Scientific Research and Technology Development Program of Nanning City (20153089, 20163127, 20173021-2), the Scientific Research and Technology Development Program of Nanning City Qingxiu District (2015S09), and the Youth Science Foundation of Guangxi Medical University (GXMUYSF2014048)

Hong-Mian Li developed the research design, evaluated all the results, and is responsible for the article. Zhi-Jie Liang and Xiang Lu developed the experimental design and analyzed the data. Dequan Li, Yi-Dan Liang, Dan-dan Zhu, and Fang-xiao Wu performed the experiments and contributed to writing the paper. Xiao-lin Yi, Ning He, Yan-Qing Huang, and Chao Tang performed clinical research.

\section{Disclosure Statement}

No conflicts of interests exists.

\section{References}

$>1$ Sinno S, Wilson S, Brownstone N, Levine SM: Current thoughts on fat grafting: Using the evidence to determine fact or fiction. Plast Reconstr Surg 2016;137:818-824.

-2 Coleman SR, Katzel EB: Fat grafting for facial filling and regeneration. Clin Plast Surg 2015;42:289-300.

-3 Agrawal KS, Bachhav M, Naik CS, Tanwar H, Sankhe SS: Autologous fat transfer for esthetic contouring of face in posttraumatic nonfunctional maxillofacial deformities. Craniomaxillofac Trauma Reconstr 2016;9:113-120.

4 Tonnard P, Verpaele A, Peeters G, Hamdi M, Cornelissen M, Declercq H: Nanofat grafting: basic research and clinical applications. Plast Reconstr Surg 2013;132:1017-1026.

5 Charles-de-Sá L, Gontijo-de-Amorim NF, Maeda-Takiya C, Borojevic R, Benati D, Bernardi P, Sbarbati A, Rigotti G: Antiaging treatment of the facial skin by fat graft and adipose-derived stem cells. Plast Reconstr Surg 2015;135:999-1009.

6 Xu FT, Liang ZJ, Li HM, Peng QL, Huang MH, Li de Q Liang YD, Chi GY, Li DH, Yu BC, Huang JR: Ginsenoside Rg1 and platelet-rich fibrin enhance human breast adipose-derived stem cell function for soft tissue regeneration. Oncotarget 2016;7:35390-35403.

7 Keyhan SO, Hemmat S, Badri AA, Abdeshahzadeh A, Khiabani K: Use of platelet-rich fibrin and platelet-rich plasma in combination with fat graft: which is more effective during facial lipostructure? J Oral Maxillofac Surg 2013;71:610-621.

-8 Cameli N, Mariano M, Cordone I, Abril E, Masi S, Foddai ML: Autologous pure platelet-rich plasma dermal injections for facial skin rejuvenation: clinical, instrumental, and flow cytometry assessment. Dermatol Surg. 2017;43:826-835.

-9 Tatullo M, Marrelli M, Cassetta M, Pacifici A, Stefanelli LV, Scacco S, Dipalma G, Pacifici L, Inchingolo F. Platelet Rich Fibrin (P.R.F.) in reconstructive surgery of atrophied maxillary bones: clinical and histological evaluations. Int J Med Sci. 2012;9:872-880.

10 Xu FT, Li HM, Yin QS, Liang ZJ, Huang MH, Chi GY, Huang L, Liu DL, Nan H: Effect of activated autologous platelet-rich plasma on proliferation and osteogenic differentiation of human adipose-derived stem cells in vitro. Am J Transl Res 2015;7:257-770. 


\section{Cellular Physiology Cell Physiol Biochem 2018;47:316-329 \begin{tabular}{lll} 
DOI: 10.1159/000489809 & and Biochemistry & $\begin{array}{l}\text { O 2018 The Author(s). Published by S. Karger AG, Basel } \\
\text { www.karger.com/cpb }\end{array}$ \\
\cline { 2 - 3 }
\end{tabular} \\ Li et al.:Skin Rejuvenation Function of Nanofat-Derived Stromal Cells and Platelet-Rich}

Fibrin

11 Cui SE, Li HM, Liu DL, Nan H, Xu KM, Zhao PR, Liang SW: Human breast adipose-derived stem cells: characterization and differentiation into mammary gland like epithelial cells promoted by autologous activated platelet-rich plasma. Mol Med Rep 2014;10:605-614.

12 Erol 00, Agaoglu G: Facial rejuvenation with staged injections of cryopreserved fat and tissue cocktail: clinical outcomes in the past 10 years. Aesthet Surg J 2013;33:639-653.

13 Chou CK, Lee SS, Lin TY, Huang YH, Takahashi H, Lai CS, Lin SD, Lin TM: Micro-autologous fat transplantation (MAFT) for forehead volumizing and contouring. Aesthetic Plast Surg 2017;41:845-855.

14 Karaaltin MV, Akpinar AC, Baghaki S, Akpinar F: Treatment of "en coup de sabre" deformity with adiposederived regenerative cell-enriched fat graft. J Craniofac Surg 2012;23:e103-e105.

15 Nicoli F, Chilgar RM, Sapountzis S, Lazzeri D, Sze-Wei MY, Ciudad P, Nicoli M, Lim SY, Chen PY, Constantinides J, Chen HC: Lymphedema fat graft: an ideal filler for facial rejuvenation. Arch Plast Surg 2014;41:588-593.

-16 Niţă AC, Jianu DM, Florescu IP, Filipescu M, Cobani O, Jianu SA, Chiriţă DA, Bold A: The synergy between lasers and adipose tissues surgery in cervicofacial rejuvenation: histopathological aspects. Rom J Morphol Embryol 2013;54:1039-1043.

17 Klar A, Güven S, Zimoch J, Zapiórkowska NA, Biedermann T, Böttcher-Haberzeth S, Meuli-Simmen C, Martin I, Scherberich A, Reichmann E, Meuli M: Characterization of vasculogenic potential of human adiposederived endothelial cells in a three dimensional vascularized skin substitute. Pediatr Surg Int 2016;32:1727.

18 Lee SH, Jin SY, Song JS, Seo KK, Cho KH: Paracrine effects of adipose derived stem cells on keratinocytes and dermal fibroblasts. Ann Dermatol 2012;24:136-143.

19 Moon KM, Park YH, Lee JS, Chae YB, Kim MM, Kim DS, Kim BW, Nam SW, Lee JH: The effect of secretory factors of adipose-derived stem cells on human keratinocytes. Int J Mol Sci 2012;13:1239-1257.

20 Banyard DA, Salibian AA, Widgerow AD, Evans GR: Implications for human adipose-derived stem cells in plastic surgery. J Cell Mol Med 2015;19:21e30.

21. Cai X, Xie J, Yao Y, Cun X, Lin S, Tian T, Zhu B, Lin Y: Angiogenesis in a 3D model containing adipose tissue stem cells and endothelial cells is mediated by canonical Wnt signaling. Bone Res 2017;5:17048.

22 Suga H, Glotzbach JP, Sorkin M, Longaker MT, Gurtner GC: Paracrine mechanism of angiogenesis in adiposederived stem cell transplantation. Ann Plast Surg 2014;72:234-241.

-23 Khan S, Villalobos MA, Choron RL, Chang S, Brown SA, Carpenter JP, Tulenko TN, Zhang P: Fibroblast growth factor and vascular endothelial growth factor play a critical role in endotheliogenesis from human adiposederived stem cells. J Vasc Surg 2017;65:1483-1492.

24 Kosaraju R, Rennert RC, Maan ZN, Duscher D, Barrera J, Whittam AJ, Januszyk M, Rajadas J, Rodrigues M, Gurtner GC: Adipose-derived stem cell-seeded hydrogels increase endogenous progenitor cell recruitment and neovascularization in wounds. Tissue Eng Part A 2016;22:295-305.

25 Li L, Pan S, Ni B, Lin Y: Improvement in autologous human fat transplant survival with SVF plus VEGF-PLA nano-sustained release microspheres. Cell Biol Int 2014;38:962-970.

-26 Dong Z, Fu R, Liu L, Lu F: Stromal vascular fraction (SVF) cells enhance long-term survival of autologous fat grafting through the facilitation of M2 macrophages. Cell Biol Int 2013;37:855-859.

27 Gir P, Oni G, Brown SA, Mojallal A, Rohrich RJ: Human adipose stem cells: Current clinical applications. Plast Reconstr Surg 2012;129:1277-1290.

-28 Gentile P, Orlandi A, Scioli MG, Di Pasquali C, Bocchini I, Cervelli V: Concise review: Adipose-derived stromal vascular fraction cells and platelet-rich plasma: Basic and clinical implications for tissue engineering therapies in regenerative surgery. Stem Cells Transl Med 2012;1:230-236.

29 Trivisonno A, Rossi A, Monti M, Di Nunno D, Desouches C, Cannistra C, Toietta G: Facial skin rejuvenation by autologous dermal microfat transfer in photoaged patients: Clinical evaluation and skin surface digital profilometry analysis. J Plast Reconstr Aesthet Surg 2017;70:1118-1128.

30 Tamburino S, Lombardo GA, Tarico MS, Perrotta RE: The role of nanofat grafting in vulvar lichen sclerosus: A preliminary report. Arch Plast Surg 2016;43:93-95.

31 Kemaloğlu CA: Nanofat grafting under a split-thickness skin graft for problematic wound management. Springerplus 2016;5:138.

-32 Xu FT, Li HM, Zhao CY, Liang ZJ, Huang MH, Li Q, Chen YC, Chi GY: Characterization of chondrogenic gene expression and cartilage phenotype differentiation in human breast adipose-derived stem cells promoted by ginsenoside Rg1 in vitro. Cell Physiol Biochem 2015;37:1890-1902. 


\section{Cellular Physiology Cell Physiol Biochem 2018;47:316-329}

\begin{tabular}{ll|l} 
DOI: 10.1159/000489809 & $\begin{array}{l}\text { O } 2018 \text { The Author(s). Published by S. Karger AG, Basel } \\
\text { www.karger.com/cpb }\end{array}$ \\
\hline
\end{tabular}

Li et al.:Skin Rejuvenation Function of Nanofat-Derived Stromal Cells and Platelet-Rich Fibrin

-33 Lo-Furno D, Tamburino S, Mannino G, Gili E, Lombardo G, Tarico MS, Vancheri C, Giuffrida R, Perrotta RE: NANOFAT 2.0: experimental evidence for a fat grafting rich in mesenchymal stem cells. Physiol Res 2017;66:663-671.

34 Abuaf OK, Yildiz H, Baloglu H, Bilgili ME, Simsek HA, Dogan B: Histologic evidence of new collagen formulation using platelet rich plasma in skin rejuvenation: A prospective controlled clinical study. Ann Dermatol 2016;28:718-724.

-35 Gawdat HI, Tawdy AM, Hegazy RA, Zakaria MM, Allam RS: Autologous platelet-rich plasma versus readymade growth factors in skin rejuvenation: A split face study. J Cosmet Dermatol 2017;16:258-264.

-36 Cho JW, Kim SA, Lee KS: Platelet-rich plasma induces increased expression of G1 cell cycle regulators, type I collagen, and matrix metalloproteinase-1 in human skin fibroblasts. Int J Mol Med 2012;29:32-36.

37 Liu B, Tan XY, Liu YP, Xu XF, Li L, Xu HY, An R, Chen FM: The adjuvant use of stromal vascular fraction and platelet-rich fibrin for autologous adipose tissue transplantation. Tissue Eng Part C Methods 2013;19:1-14.

38 Leach JB, Bivens KA, Collins CN, Schmidt CE: Development of photocrosslinkable hyaluronic acidpolyethylene glycol-peptide composite hydrogels for soft tissue engineering. J Biomed Mater Res A 2004;70:74-82.

-39 Ulusal BG: Platelet-rich plasma and hyaluronic acid - an efficient biostimulation method for face rejuvenation. J Cosmet Dermatol 2017;16:112-119. 